\title{
Human Behavioral Simulation Using Affordance-Based Agent Model
}

\author{
Namhun Kim ${ }^{1}$, Jaekoo Joo ${ }^{2}$, Ling Rothrock ${ }^{3}$, Richard Wysk ${ }^{4}$, and Young-Jun Son ${ }^{5}$ \\ ${ }^{1}$ Design and Human Engineering, Ulsan National Institute of Science \\ and Technology, Republic of Korea \\ ${ }^{2}$ Systems and Management Engineering, Inje University, Republic of Korea \\ ${ }^{3}$ Industrial and Manufacturing Engineering, the Pensylvania State University, \\ University Park, PA 16802, USA \\ ${ }^{4}$ Industrial and Systems Engineering, North Carolina State Universty, \\ Raleigh, NC 27695, USA, and \\ ${ }^{5}$ Systems and Industrial Engineering Department, The University of Arizona, \\ Tucson, AZ 85721, USA \\ nhkimaunist.ac.kr
}

\begin{abstract}
In this paper, we propose a novel agent-based simulation modeling of human behaviors. A conceptual framework of human behavioral simulation is suggested using the ecological definition of affordances in order to mimic perception-based human actions interacting with the environment. A simulation example of a 'warehouse fire evacuation' is illustrated to demonstrate the applicability of the proposed framework. The perception-based human behaviors and planning algorithms are adapted and embedded within human agent models using the Static and Dynamic Floor Field Indicators, which represent the evacuee's prior knowledge of the floor layout and perceivable information of dynamic environmental changes, respectively. The proposed framework is expected to capture the natural manners in which humans participate in systems and enhance the simulation fidelity by incorporating cognitive intent into human behavior simulations.
\end{abstract}

Keywords: Human Behavior, Affordance Theory, Finite State Automata, Agent-based Modeling, and Simulation.

\section{Introduction}

While systems theory has grown rapidly, modeling and simulation of human-involved systems has not kept pace. We suspect that it is, in part, because no common framework exists to accommodate both human cognitive models and the discrete system representations within which humans play critical roles. This creates a major modeling void as most complex systems contain human activities. In this research, we present a generic simulation framework to fill up the void using the ecological concept of affordance and a formal modeling methodology for human-involved complex systems.

In 1979, Gibson initiated an ecological understanding of human actions in animalenvironment systems [1]. According to him, a human action is regarded as a consequence 
of the juxtaposition process of affordance and effectivity (an individual's property of ability to take a specific action) which are directly perceived by the human. That means a human makes decisions to take actions based on perceived information regarding sets of affordance-effectivity couples.

From this perspective, we run into the challenge to address the human in the system and to provide a modeling vision for a human-involved system. In this research, an affordance-based simulation framework that is capable of analyzing perception-based human behavioral patterns in complex and uncertain environs is developed for modeling systems with people. For illustrative purposes, we discuss the problem from the standpoint of the ecological concept of affordance to interpret human actions and simulate their perception-based planning and behavioral patterns. The proposed framework will provide a predictive analysis capability for the design of human-involved system.

The remainder of this paper is organized as follows: Section 2 provides the related work and background of this research. Section 3 presents the conceptual simulation framework and its components to cater for human-involved discrete event simulations. We demonstrate the proposed simulation framework by implementing the 'ware house fire evacuation' scenario in which human evacuees make decision based on their perception as well as previous knowledge in Section 4. Finally, we conclude this paper with a discussion of the direction for future research in Section 5.

\section{Background}

\subsection{Human Behavior Modeling and Simulation}

Human decision behaviors have been studied by researchers in various disciplines such as artificial intelligence, psychology, cognitive science, and decision science. Lee et al. [2] classified human decision behavior models into three major categories based upon their theoretical approach: 1) economics-based approach, 2) a psychology-based approach, and 3) a synthetic engineering-based approach. Each approach exhibits strengths and limitations. First, models employing the economicsbased approach have a concrete foundation, based largely on the assumption that decision makers are rational [3, 4]. However, one limitation is the inability to represent the nature of human cognition. To overcome this limitation, models using a psychology-based approach have been proposed [5,6]. While these models explicitly account for human cognition, they generally address human behaviors only under simplified and controlled laboratory conditions [7].

While current synthetic models available in the literature consider human perceptions explicitly, none of them is grounded both on the ecological concept of affordance and a formal system theoretic method for human-involved complex systems. Therefore, the goal of this research is to develop a generic framework for affordance-based simulation of human behavior using affordance-based finite state automata (FSA). 


\subsection{Affordance Theory and Perception-Based Human Actions}

Gibson first defined the affordances of the environment as what it offers to the animal (person), what it provides or furnishes, either for good or ill [1]. Since Gibson proposed his definition, the notion of affordance has been further refined and theorized. For example, Turvey presents a perspective on the ecological ontology of affordances with links to prospective control [8]. Turvey bases the definition of affordance in terms of properties that represent a potential state and are not currently realized (called dispositional properties or dispositions). Dispositions occur in pairs in which a property of the environment (i.e., walk on - ability for the person) is complemented by a property of the animal's capability known as an effectivity (i.e., to walk on the stairs' surface). So the terms of affordance and effectivity can be combined together so that they incur a different property (i.e., climb the stairs) to be activated [8]. Specifically, Turvey presents a formal definition of affordances mathematically using a juxtaposition function as follows;

Let $W_{p q}=j\left(X_{p}, Z_{q}\right)$ be a function that is composed of an animal $(X)$ and a environmental object $(Z)$, and further $\mathrm{p}$ and $\mathrm{q}$ be properties of $X$ and $Z$, respectively. Then, $p$ refers to an affordance of $X$ and $q$ is the effectivity of $Z$, if and only if there exists a third property $r$ such that:

i) $W_{p q}=j\left(X_{p}, Z_{q}\right)$ possesses $r$,

ii) $\quad W_{p q}=j\left(X_{p}, Z_{q}\right)$ possesses neither $p$ nor $q$, and

iii) Neither $X$ nor $Z$ possesses $r$, where $r$ is a joining or juxtaposition function.

For example, in case of a person-climbing-stairs system $(W)$, a person $(Z)$ can walk $(q)$, stairs $(X)$ can support something $(p)$, and they together yield climbing property $(r)$. This formal definition of affordance, effectivity, and juxtaposition function can be mapped to the precondition set of state transition function in the FSA and provides a foundation that the concept of an affordance can be combined with software engineering and systems theory.

\subsection{Affordance-Based Formal Modeling of Perception-Based Human Actions}

The affordance-based modeling approach has been widely adapted for designing robot controls and mimicking human actions in specific environmental situations [9]. However, most of these efforts lack formal ways to model human actions with respect to system transitions, due to the low level of abstraction in modeling perceptual properties.

Kim et al. suggest an affordance-based descriptive formalism for human-involved complex systems using finite state automata. In their work, an environmental system is defined as a set of nodes and arcs which describe discrete states of the system and the transitions between states, respectively [10]. Then, a set of transitions between states is triggered by certain human actions to lead to the next states. Affordanceeffectivity combinations are considered as preconditions for actualizing possible human actions. In their work, a perception-based human action and its associated properties of an affordance and effectivity are systematically defined as placeholders in the context of discrete systems for implementation of a dynamic juxtaposition process (see Figure 1). There are two kinds of state transitions: external state transition and internal state transition. The external state transition can bring physical 
changes of system state caused by a specific human action in the human-environment system. On the other hand, the internal state transition is concerned with a precondition(s) that must be satisfied for actuating the external state transition in the system. It connects two sub-states each of which is a combination of a specific affordance and effectivity. These affordances and effectivities are time-varying properties which are determined by the physical situations of environmental elements and human capabilities. In a certain amount of time, $t_{\text {int }}$, if the current status of affordances and effectivities $\left(p_{i}, q_{j}\right)$ may be changed to $\left(p_{m}, q_{n}\right)$ to meet a specific action condition, then the juxtaposition function will generate a human action and then an external state transition will occur, and then a physical state of the system goes to the next. The formal modeling of human behaviors in the sense of perceptionbased decision making provides a systematic way to include physical preconditions of human action possibilities within computable modeling methodologies.

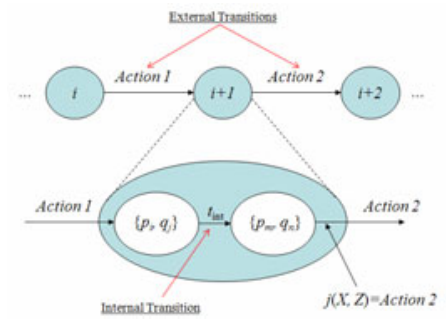

Fig. 1. External and internal transitions of Affordance-FSA formalism [10]

\section{Conceptual Framework of Affordance-Based Human Behavior Simulation}

\subsection{System Architecture of Affordance-Based Simulation}

In the system theoretic perspective, a human can be regarded as a non-deterministic and unpredictable agent within an environment. Envision of theory of affordance allows us to include the properties of perception-based actions into discrete eventbased systems and to build the affordance-based simulation framework [10]. The proposed affordance-based simulation framework is able to evaluate action possibilities and predict human behaviors using the embedded formal automata models of human-involved systems. It considers both affordance and effectivity as dynamic control parameters which trigger human actions in the simulation in order to deal with the interactions between human and dynamic environmental elements.

Figure 2 depicts the system architecture of the proposed affordance-based simulator, where four major components include 1) Affordance-based FSA model, 2) Agent Models, 3) Event Generator, and 4) Human Action Planner. The Affordancebased FSA models are formal automata models of human-involved systems that describe the whole state map of human actions and dynamics of environments. The FSA model provides dynamic (temporal and spatial) situations and preconditions of possible transitions for agents in the system. The agent models are classified into a 
human agent and an environmental agent. A human agent represents each human in the system. An environmental agent describes each environmental element (or cue) of the system which may affect human decision makings in the simulation model such as fire, smoke, and flood. Each human agent has an action planning (or decision) algorithm and the environmental agent follows its own governing rule based on physics, while they make transitions from one state to another on the state map of the affordance-based FSA. The event generator triggers each event according to predefined human action plans by perceived property data of affordances and effectivities from a human agent. The human action planner generates a feasible sequence of actions reaching from the current toward the goal state for a human agent. In order to do that, the planner refers to dynamic data of affordances and effectivities as well as static data of human prior knowledge such as the layout of an environmental space. If an unexpected or undesired situation occurs in the system, it causes a transition that leads to deviation from the active plan. If it happens, the planner immediately recalculates the plan in order to cope with the dynamic change of the environment.

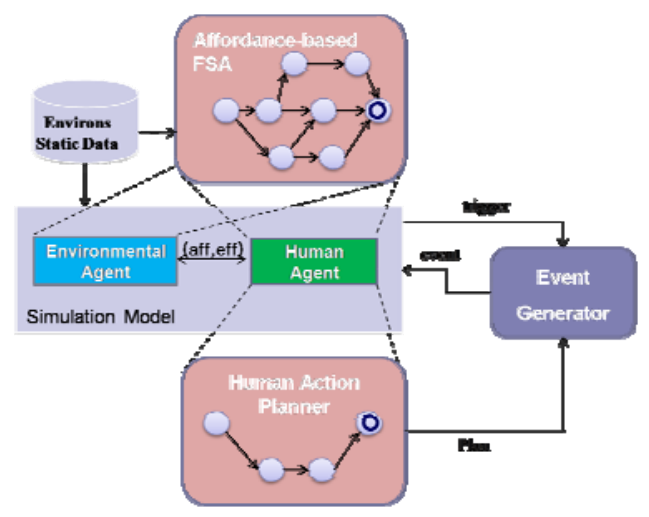

Fig. 2. System architecture of the proposed affordance-based human behavior simulator

\subsection{Agent Models}

The concept of agent can vary by its application area. In software engineering, the most widely accepted definition of agent model is that: the agent is a computer system that is situated in some environment and capable of autonomous action to meet the system objectives [11]. An agent in simulation model implies the nature of each entity and expresses the complex interaction with other agent in the environment, so that the simple agent rule can generate the complex system behaviors.

In this research, two different types of agent models are defined for the model verification (see Figure 3). While a human agent model is represented by goals, perception abilities, decision making algorithm and action capabilities, an environmental agent maps dynamics of environmental elements onto the system model. The concepts of perception-based human action and planning are used to develop the decision making algorithm in a human agent model in Section 3.3. 
Several attributes (e.g. type, name, moving speed, etc) and characteristics for each agent are defined to reflect the diversification of humans and environmental elements in the system. A conceptual model is developed to illustrate functional procedures of and interactions between the human agent and the environmental agent in Figure 4. It is noteworthy that human actions are affected by the information on affordances and effectivities perceived in the human-environment system.

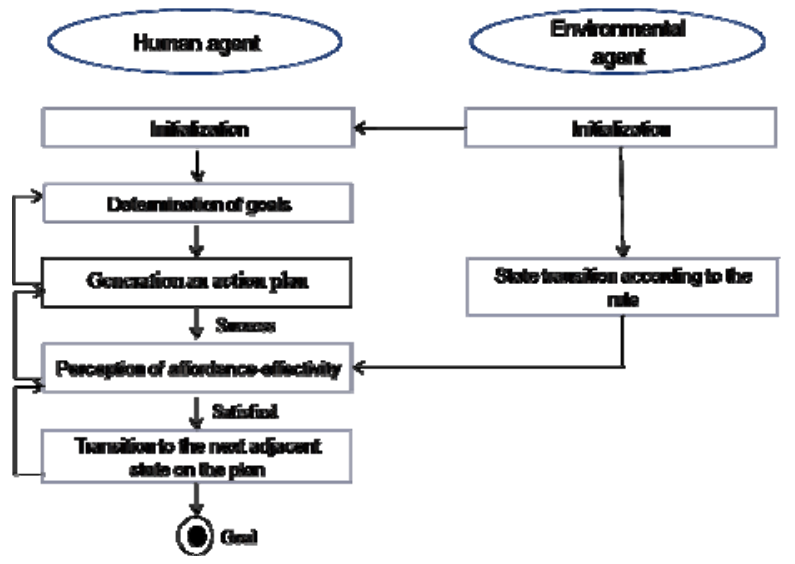

Fig. 3. Conceptual model of human agent and environmental agent

\subsection{Human Action Planner}

Considering the perception based actions and planning of human agents, we need to define a concept of a perceivable boundary (PB) as a horizontal space that a human can visually perceive surrounding situations. Since perceivable boundary is determined by the position of a person and dynamics of other environmental conditions such as lighting and fog, it should be continuously updated according to the movements of a person. While the shape and range of a perceivable boundary are dependent on the human visual direction, it is assumed in this example that human agents have 360 degree viewing angle by rotating their body and head of their own free wills.

The plan is generated based on not only static information of human knowledge on the surface layout of an environment, but also perception of dynamic information within perceivable boundary obtained at the very moment of the planning (or replanning) and decision making. In this research, the static information is defined as Static Floor Field Indicator (SFFI) which is a set of numbers indicating distance to the exit [12], whereas the dynamic information as Dynamic Floor Field Indicator (DFFI) which is a set of numbers representing affordances (move-abilities for a human) of perceived areas within a perceivable boundary.

For the static information in the simulation, it is assumed that a human has previous knowledge about the floor layout regardless of his or her perception of the environment, so as to make a complete plan which persists until achieving his goal. He or she is supposed to know how far the goal position (i.e., the exit) is from the 
current position in the layout. For the dynamic and complex situation which can change the floor layout, however, it is not the case. Suppose that the evacuation route for human should be changed because of a fire on a corridor within the evacuee's original evacuation route. If the system is too large for a person to perceive the entire area, he or she cannot know every details of a current situation of the environment beyond his or her PB. So, the evacuee with insufficient information of the environment can make his or her evacuation plan only within the perceivable boundary. Beyond the boundary, he or she may be able to make a rough plan with the layout information in his or her memory. The rough plan here means a plan made only with prior knowledge of the layout (static information). On the other hand, the detailed plan is made based on both the static information (prior knowledge) as well as dynamic information (perceptual properties) within the perceivable boundary. The planner generates and updates both types of plans every time it's needed (e.g, when an unexpected situation which blocks the current planning path occurs). While the detailed plan within a perceivable boundary is expressed via affordance-based FSA considering perceptual properties of affordances and effectivities, the rough plan outside of the perceivable boundary is generated based on the FSA model without consideration of the perceptual properties.

The evacuee decides his or her movement to next position based-on his or her objective of behavior in the system which is composed of conditions and goals. An exemplary objective of human behavior is "safe and quick (conditions) escape to an exit (goal)" in the case of emergency evacuations. In this paper, we propose a generic planning algorithm that can be easily applied to generate a human action plan for the affordance-based simulation problem as follow:

- Step1: Define current location of a human agent and perceivable boundary (PB).

- Step2: Based on the current information, make an action plan until the final states (goal state) by calling an objective analysis algorithm. If multiple solutions exist, then randomly choose one of them. (It should be noted that the objective analysis algorithm can be developed for each application domain by specifying its characteristics.)

- Step3: Move the human agent to the next position on the plan as long as the position is currently affordable. If the human agent reaches the final state (goal state), then stop.

- Step4: Update PB and FFIs of cells that newly added to PB. If any state in the plan is not affordable, go to Step 2 and revise the plan. Otherwise, go to Step3.

\section{Illustration: Warehouse Fire Evacuation Problem}

\subsection{Scenario}

To verify the applicability of the proposed framework of affordance-based simulation, the Warehouse Fire Evacuation (WFE) problem is investigated in this section. In this considered WFE Problem (see Figure 4-(a)), a fire breaks out in a warehouse in which two human operators are working. The warehouse area is equally divided in a rectangular grid of $0.8 \times 0.8 \mathrm{~m}^{2}$ which is used for either storage or passageway. In this storage area, goods are stacked up so high that operators cannot observe what happens 
over the storing lots. Fires are firstly broken out at three different locations in the warehouse and are fast propagated to neighboring lots in a certain amount of speed as shown in Figure 4. As soon as an operator perceives the fire, he/she shall have to find a possible and safe route to an exit along passageway by considering perceived surrounding situations in order to escape from the fires. When he/she tries to move to a next passageway lot, if the lot is already occupied with a fire, he/she cannot access to the lot and have to find another passageway that offers an affordance to move. The following assumptions are made to simplify the considered problem:

1. Each evacuee can perceive the situations with 360 degree viewing angle in the warehouse.

2. An evacuee can move to next lots only in the direction of front and back or right and left in right angle. He/she cannot move to diagonal directions in the warehouse.

3. Every evacuee acts independently with each other, but some interactions are possible among evacuees to share their information and knowledge about the floor layout or current situations of the warehouse when they meet in the same places.

4. Fire is only considered as dynamic environmental element. (Smoke and heat are excluded.)

\subsection{Model Description}

The human behaviors in this evacuation problem can be interpreted as a typical example of affordance-based human actions in prospective control. Turvey defines that prospective control is concerned with future events, usually interpretable as goals to be realized [8]. In this example, each evacuee determines his or her own goal and plan before starting to move. The goal of each evacuee is to get out of the warehouse via one of the exits while avoiding a spreading fire. Once a goal is determined, each evacuee will make a plan for his or her movements along passageways to the exit. To this end, the plan is made by using not only his or her prior knowledge about the floor layout in the warehouse, but perceived information on surrounding situations within the $\mathrm{PB}$ at the moment of the planning. A concept of the goal and plan in this evacuation problem is also found in Figure 4-(a).

In order to realize this evacuation problem into a simulation model, the warehouse is described by a two-dimensional grid of equally divided 64 lots. Each cell can be empty, occupied by goods or by fires. Its size corresponds to $0.8 \times 0.8 \mathrm{~m}^{2}$, the typical surface occupied by more than two persons in a dense situation [13]. Once the geometry of the floor field and exit locations is determined, each cell is assigned with a constant value of a static floor field indicator (SFFI) representing its static distance to the exits. Nearer the exit, lower values correspond to cells. Thus, these static values indicate to the evacuees the direction to the exit, since they always prefer to move to an adjacent cell with a lower value of SFFI than the current one. So the values of the floor field indicator are used as a basis for generating plans to the way to the exits with dynamic perceptual information on the floor. Cells occupied by goods or fires are assigned with very high values of dynamic floor field indicators (DFFIs) to ensure that there is no affordance of move-ability to an evacuee and he or she will never attempt to occupy them. The floor layout and the values of the static floor field indicators are shown in Figure 4-(a). 


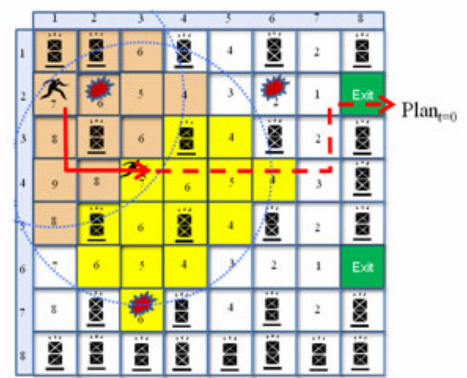

(a) human action plan and PB at time $t_{0}$

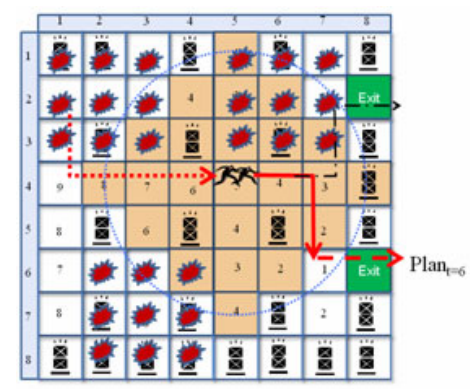

(b) human action plan and PB at time $t_{6}$

Fig. 4. Entity state transition model for Warehouse Fire Evacuation Problem

In the affordance-based simulation model, each evacuee is represented as a human agent, and fire is represented as an environmental agent. The continuous time is discredited into time steps which are unit times for the simulation. The mean velocity of evacuees is around $2 \mathrm{~m} / \mathrm{s}$ [14]. If we set the time step $\Delta$ t to $0.4 \mathrm{~s}$, human agents move $0.8 \mathrm{~m}$ per time step in average. The mean velocity of fire propagation is assumed to be $0.4 \mathrm{~m} / \mathrm{s}$. This means that a fire broken out in a cell can be propagated into its adjacent cells at every five time steps averagely. Therefore dynamics of the warehouse can be implemented by the floor layout of the warehouse and a dynamic interaction between human agents and propagating fire agents in the warehouse.

To perform this behavior, each human agent perceives whether its adjacent lots provide it with the affordance, "is-move-able" for the human agent, or not. So, in this warehouse evacuation problem, the affordance is "move-ability for an evacuee to an adjacent lot," and the accompanying effectivity for a human agent is "capability to move to an adjacent lot."

\section{Conclusion}

In this research, we suggest a generic framework for human behavior simulation by combining both human cognitive models and the discrete system representations, which is the affordance-based FSA. The simulation model presented in this paper is agent-based as well as perception-based. The human agent (evacuee) in the demonstrated simulation model has its own algorithm to generate evacuation plans based on both the prior knowledge on the floor layout and what it perceives in the perceivable boundary. The agent model can regenerate evacuation plans whenever its perceivable surrounding environments are changed. This simulates realistic human behaviors which are dependent on perception-based decision making claimed by ecological psychologists.

While we focused on the conceptual framework and human's perception (a major function) in this work, we will consider other human attributes (e.g. social psychology, emotions, cultures, and knowledge levels) in the future. Also, we shall have to validate the simulation results via human experiments in a suitable task environment (e.g. virtual reality). 
Acknowledgment. This research was supported by Basic Science Research Program through the National Research Foundation of Korea(NRF) funded by the Ministry of Education, Science and Technology(20090077993) "

\section{References}

1. Gibson, J.J.: The Ecological Approach to Visual Perception. Houghton Mifflin, Boston (1979)

2. Lee, S., Son, Y., Jin, J.: Decision field theory extensions for behavior modeling in dynamic environment using Bayesian belief network. Information Sciences 178(10), 2297-2314 (2008)

3. Mosteller, F., Nogee, P.: An experimental measurement of utility. J. Pol. Econ. 59, 371404 (1951)

4. Opaluch, J.J., Segerson, K.: Rational roots of irrational behavior: New theories of economic decision-making. Northeastern Journal of Agricultural and Resource Economics 18(2), 81-95 (1989)

5. Edwards, W.: The theory of decision making. Psychological Bulletin 51(4), 380-417 (1954)

6. Payne, J.W.: Contingent decision behavior. Psychological Bulletin 92, 382-402 (1982)

7. Rothrock, L., Yin, J.: Integrating compensatory and noncompensatory decision making strategies in dynamic task environments. In: Kugler, T., et al. (eds.) Decision Modeling and Behavior in Complex and Uncertain Environments, pp. 123-138. Springer, New York (2008)

8. Turvey, M.T.: Affordances and Prospective Control: An Outline of the Ontology. Ecological Psychology 4(3), 173-187 (1992)

9. Kirlik, A., Miller, R.A., Jagacinski, R.J.: Supervisory Control in a Dynamic Uncertain Environment: A Process Model of Skilled Human-Environment Interaction. IEEE Transactions on Systems, Man, and Cybernetics 23(4), 929-952 (1993)

10. Kim, N., Thiruvengada, H., Rothrock, L., Wysk, R.A., Shin, D.: Modeling of Affordances in Human-involved Complex Systems Using Finite State Automata (FSA). IEEE Transactions on Systems, Man, and Cybernetics Part A: Humans and Systems (submitted for publication)

11. Varas, A., Cornejo, M.D., Mainemer, D., Toledo, B., Rogan, J., Munoz, V., Valdivia, J.A.: Cellular automaton model for evacuation process with obstacles. Physica A: Statistical Mechanics and its Applications 382, 631-642 (2007)

12. Zhang, Z., Zhang, C.: Agent-Based Hybrid Intelligent Systems: An Agent-Based Framework for Complex Problem Solving. Springer, New York (2004)

13. Burstedde, C., Klauck, K., Schadschneider, A., Zittartz, J.: Simulation of Pedestrian Dynamics using a two-dimensional cellular automaton. Physica A: Statistical Mechanics and its Applications 295, 507-525 (2001)

14. Daoliang, Z., Lizhong, Y., Jian, L.: Exit dynamics of occupant evacuation in an emergency. A: Statistical Mechanics and its Applications 363, 501-511 (2005) 